\title{
Prediction of flow induced vibrations in vertical turbine pumps using one-way fluid-structure interaction
}

\author{
Ravindra Birajdar ${ }^{1, *}$ and Appasaheb Keste $^{2}$ \\ ${ }^{1}$ Kirloskar Brothers Limited, Corporate Research \& Engineering Development, Pune, Maharashtra, \\ India \\ ${ }^{1}$ Trinity College of Engineering \& Research, Department of Mechanical Engineering, Pune, \\ Maharashtra, India \\ ${ }^{2}$ MES College of Engineering, Department of Mechanical Engineering, Pune, Maharashtra, India
}

\begin{abstract}
Centrifugal pumps/vertical turbine pumps are important and critical equipment for operation in the power generation, water supply, process industries and petrochemical industries. All centrifugal and vertical turbine pumps have rotors and structures that can vibrate in response to excitation forces. Mechanical and hydraulic unbalance are the two major factors which can create dynamic effects in terms of pump vibrations. In many cases the resulting hydraulic forces due to hydraulic unbalance has much the same effect as the mechanical unbalance. The vibrations in the pumps must be within acceptable limits of applicable standards. If there is higher level of vibrations, it not only leads to operational inefficiencies but also causes pump failures. Hence, it is very important for designers to understand the dominating fact of unbalance force and its origin. The prediction of vibrations in a vertical turbine pump due to the hydro dynamic forces, using numerical methods can help a designer to accomplish a successful design. The interaction between solid and fluid in present case can be completed by one-way coupling method. The one-way fluid-structure interaction approach is presented in present paper to predict the vibrations at specific operating condition which has good correlation with the test data. The advantage of reduced computational effort in this approach can be utilized during initial design stage. In this paper, a case study of one-way FSI approach of a vertical turbine pump is discussed. After detailed explanation about the CFD results, one-way coupling approach is explained with comparison of vibration displacements in both numerical and test data.
\end{abstract}

\section{Introduction}

Vertical turbine pumps have extensive applications in the industry. The major applications include in Irrigation projects, cooling water circulation pumps in power plants. The limited space utilization for handling the large amount of fluid and simplified piping for submerged

${ }^{1}$ Corresponding author: ravindra.birajdar@kbl.co.in 
pumping requirements made them very economic compared to other pumps. Vibrations in pumps may be a result of improper installation or maintenance, incorrect application, hydraulic interaction with the piping system, or design and manufacturing defects. All centrifugal and vertical turbine pumps have rotors and structures that can vibrate in response to excitation forces. Some of the main causes of excessive pump vibrations and failures are given below in Table 1 [1].

Table 1. Causes of excessive pump vibrations

\begin{tabular}{|l|l|l|}
\hline \multicolumn{1}{|c|}{ Rotor dynamic } & \multicolumn{1}{c|}{ Fluid Dynamic } & \multicolumn{1}{c|}{ Structural } \\
\hline $\begin{array}{l}\text { Pump lateral critical } \\
\text { speeds }\end{array}$ & Hydraulic Instabilities & $\begin{array}{l}\text { Bearing housing / } \\
\text { pedestal resonances }\end{array}$ \\
\hline Torsional critical speeds & Recirculation & $\begin{array}{l}\text { Case distortions caused } \\
\text { by piping strain }\end{array}$ \\
\hline Seal Rubs & $\begin{array}{l}\text { Pressure pulsations and } \\
\text { acoustical resonances }\end{array}$ & $\begin{array}{l}\text { Impeller resonances } \\
\text { Unbalance }\end{array}$ \\
$\begin{array}{l}\text { System flow distribution } \\
\text { problems }\end{array}$ & $\begin{array}{l}\text { Piping lateral Mechanical } \\
\text { Resonances }\end{array}$ \\
\hline $\begin{array}{l}\text { Coupling or shaft-to- } \\
\text { shaft misalignment }\end{array}$ & Water hammer & Foundation resonances \\
\hline Shaft Instabilities & Inadequate NPSH & $\begin{array}{l}\text { Loose tie down bolts } \\
\text { and/or Grout }\end{array}$ \\
\hline
\end{tabular}

Many of these causes are a result of an interaction of the pump with the fluid or the structure. This interactive relationship requires that the complete system be evaluated instead of investigating individual components when problems occur. Mechanical unbalance occurs when the mass centreline of a rotating component does not coincide with the shaft centreline. The unbalance force rotates with rotor speed (1X) and is therefore a sinusoidal function of time when viewed from stationary frame of reference. Geometrical deviations in the impeller channels create a non-uniform pressure distribution at impeller outlet, which also rotates with rotor speed [2]. The resulting radial hydraulic force has much the same effect as mechanical unbalance and is therefore referred to as hydraulic unbalance. Hydraulic unbalance increases with increasing flow-rate and usually exceeds mechanical unbalance.

This paper presents an approach which can be taken to analyse vertical turbine pump dynamics and investigate the effect of fluid induced forces on the pump vibrations at first glance. CFD analysis has been carried out for the vertical pump for an operating point and the induced hydraulic forces at respective flow are mapped on to the structural mesh to conduct the vibration analysis. The displacements from the numerical analysis were measured at motor location and compared with the available test data. This approach can be used to identify the source of the hydraulic unbalance for specific operating conditions, to study the effect of geometrical deviation on hydraulic unbalance and the impact of design modifications aimed to reduce the vibrations.

\section{Vibrations in vertical pumps}

The proper operation of vertical pumps is greatly depending on the total pump suction conditions, pump discharge piping system and the system operating conditions. The total pumping system of a vertical turbine pump can be divided into 3 parts (1) Pump intake (2) pumping system and (3) Discharge system [3].

(1) Pump intake describes the approach flow quality towards the pump bell mouth. Proper design of a pump intake is necessary to avoid the following flow related phenomenon like pre-swirl magnitude, free surface and submerged vortices. (ANSI/HI 9.8, 1998). 
(2) Pump generated dynamic pressure sources include vortices or wakes produced in the clearance space between impeller vane tips and the stationary bowl. Dynamic pressure fluctuations or pulsations produced in this manner can cause impeller vibrations or can result in shaft vibrations as pressure pulses impinge on the impeller.

(3) The discharge system consists of the components downstream of the pump bowl. It basically consists of pump column, shaft, discharge head and the motor. The discharge systems can respond to the flow patterns resulting by pumping system. The flow output resulting from the pumping system can act as exciting source of vibration.

Many of these causes are a result of an interaction of the pump with the fluid or the structure. This interacting relationship requires that the complete system be evaluated rather than investigating individual components when problems occur.

\section{Fluid structure interaction}

Fluid-structure interaction is a Multiphysics problem which occurs in a system where flow of fluid causes deformations in the structural system. A solid structure having a fluid flow is subjected to a pressure induced by the fluid and hence cause deformation in the structure. As a return, the deformed structure changes the flow field. The change in flow field again exerts change in pressure on the structural part. This kind of repetitive interaction is called FluidStructure Interaction [4]. In general, a fluid-structure interaction can be classified into two methods of coupling. (1) One-way coupling (2) Two-way coupling. The information exchanged is dependent on the coupling method. For one-way coupling calculations, only the fluid pressure acting at the structure is transferred to the structure solver. For two-way coupling calculations, the displacement of the structure is also transferred to the fluid solver. The present study totally focusses on the one-way coupling of the fluid and structure.

\section{Numerical analysis and vibration results}

\subsection{Problem description}

The duty parameters of vertical pump considered for the analysis are Q-30000 m3/hr, H$23.5 \mathrm{~m}, \mathrm{~N}-373 \mathrm{rpm}$. Vibration displacements at motor top surface are to be measured using numerical analysis and will be compared with the available test data. CFD analysis has been carried out for duty point and the pressure data was transferred to structural part which would act as input for the vibration analysis. This process includes the mapping of the pressure value on the wet wall surface of vertical pump to the structural part of the pump to determine the vibration displacements. Fig. 1 shows the geometry and mesh used in the analysis in successive steps. 


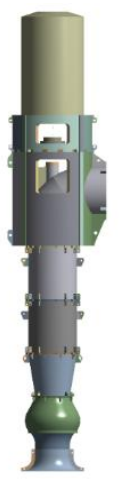

(a)

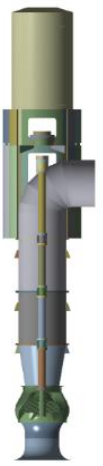

(b)

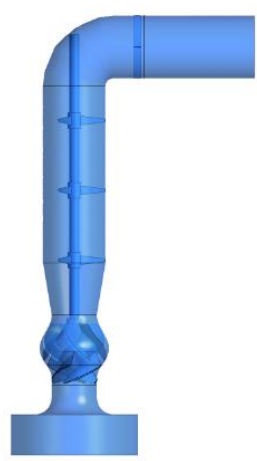

(c)

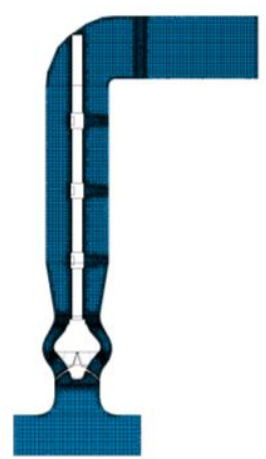

(d)

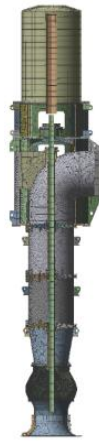

(e)

(a)Vertical pump geometry (b) Section view (c) Fluid volume (d) Finite volume mesh of fluid volume (e) Finite element mesh of structural part

Fig. 1. Three-dimensional geometry and mesh of vertical turbine pump

\subsection{CFD analysis}

The hydraulic model was extracted from the prepared mechanical model of the vertical turbine pump. In the present geometry, the uniform flow near the bell mouth is assumed. Hence the portion at inlet is extended as a circular periphery considering the limits of back wall clearance and bottom wall clearance. The boundary conditions were set in the geometry as per given flow and speed. The k- $\varepsilon$ turbulence model was used with scalable wall function, a convergence criterion of 1E-05 and high-resolution scheme.

\subsection{Structural analysis}

The structural model of pump with motor is prepared using internal wet surfaces as the driving dimensions for shell modelling to achieve pressure mapping with least possible deviation. Added mass effect of the fluid filled in pump wetted portions is considered along with additional mass of fluid displaced by the submergence of pump unit. This model is then constrained at the foundation bolt pads and discharge nozzle flange is fixed assuming piping connections to be rigid connections. Now the pressure values obtained from CFD is mapped to wet surfaces of the pump are considered as applied structural loads.

\subsection{One-way FSI coupling}

The numerical solutions are based on a partitioned approach where separate solutions for the different physical fields are prepared. The two physical fields are fluid and structure. Fluid part is solved using CFD methods and the structural part is solved using structure dynamics. The boundary between the fluid part and solid part is used as fluid-structure interface; information for the solution is shared between the fluid solver and structure solver. In present one-way coupling, the obtained pressure values on the internal walls of the hydraulic model are mapped on to the structural model. Fig.2.shows the comparison of the pressure contours both in CFD model and the structural model. The present model is found to be mapped with $5 \%$ deviation. 


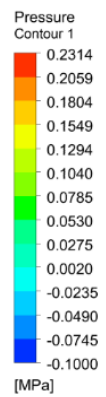

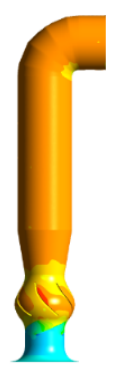

CFD Model
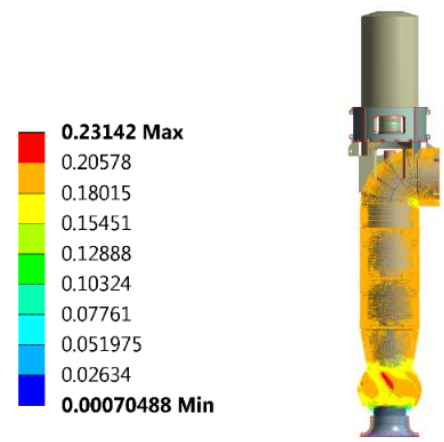

Structural model after pressure mapping

Fig. 2. Pressure mapping from CFD model to structural model

\subsection{Numerical results}

The vibration values obtained from structural analysis with above boundary conditions are compared with the test data obtained from the site. Table 2 shows the comparison of vibrational displacements from numerical simulation with the test results. The available test data consists of the displacements measured at top of the motor in 3 directions $\mathrm{H}, \mathrm{V}, \mathrm{A} . \mathrm{H}$ represents the direction perpendicular to discharge, $\mathrm{V}$ represents the direction along discharge, A represents along axis of the shaft. The vibrational displacements in V and A direction are in good correlation with test data at initial guess. The fluid induced forces are causing vibrations in direction of discharge which is $\mathrm{V}$ are well predicted with one-way FSI. The variation in $\mathrm{H}$ vibrations can be due to the mechanical reasons as it is not significantly affected by fluid induced forces. Fig.3. and 4 shows the contours of the displacements in $\mathrm{H}$, $\mathrm{V}$ and $\mathrm{A}$ direction for vertical turbine pump and motor respectively.

Table 2. Comparison of vibration displacements with test data.

\begin{tabular}{|l|c|c|c|c|c|c|}
\hline & \multicolumn{5}{|c|}{ Vibration displacements in microns $(\boldsymbol{\mu m})$} \\
\hline Component & \multicolumn{4}{|c|}{ Test data $(\boldsymbol{\mu m})$} & \multicolumn{3}{c|}{ Numerical Results $(\boldsymbol{\mu m})$} \\
\hline & $\mathrm{H}$ & $\mathrm{V}$ & $\mathrm{A}$ & $\mathrm{H}$ & $\mathrm{V}$ & $\mathrm{A}$ \\
\hline Discharge bend & - & - & - & 178.7 & - & - \\
\hline Bellmouth & - & - & - & - & 221.57 & - \\
\hline Bellmouth & - & - & - & - & - & 173.3 \\
\hline Motor vibrations & 64 & $\mathbf{6 2}$ & $\mathbf{1 5}$ & 2.57 & $\mathbf{4 9}$ & $\mathbf{1 1}$ \\
\hline
\end{tabular}

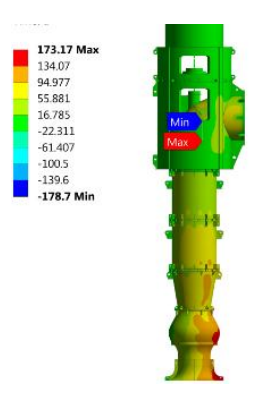

H

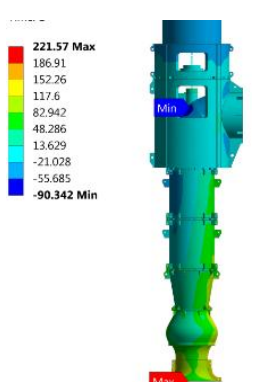

V

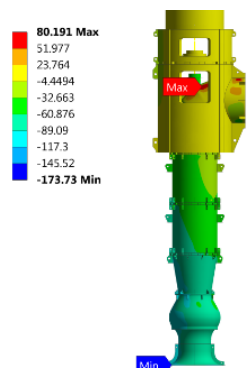

A

Fig. 3. Displacement contours on vertical turbine pump in 3 directions 

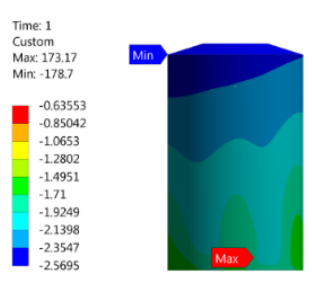

$\mathrm{H}$

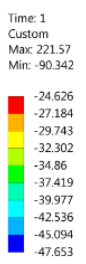

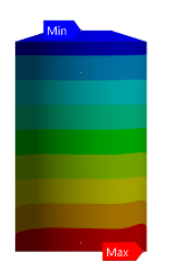

V

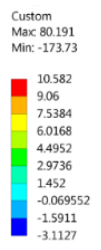

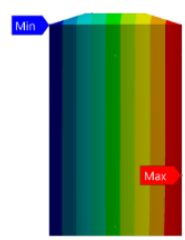

A

Fig. 4. Displacement contours on motor in 3 directions

\section{Conclusions}

In this paper, the interaction between the fluids and structures were solved with one-way coupling method to predict the flow induced vibrations in a vertical turbine pump. The pressure mapping was applied for the wetted surfaces in a vertical pump with a specific care about the direction of hydraulic load on specific parts. The vertical pump is fixed at discharge end and near the base plate as per the general fixed locations. The deviation in the mapping is found to be less than $5 \%$.

For a case with available test data, the obtained results of deflection values are found to be in good correlation with test data. Vibration displacements are measured near motor location for directions $\mathrm{H}, \mathrm{V}, \mathrm{A}$ and compared with the respective test values. Overall, the one-way coupling fluid structure interaction method in vertical pumps can be used as initial method to understand and predict the fluid induced vibrations in any vertical pump.

\section{References}

[1] J. C. Wachel, J.D. Tison, and K. E. Atkins, "Field Instrumentation and Diagnostics of Pump Vibration Problems," in Rotating Machinery and Controls (ROMAC), Virginia, June (1983).

[2] T. F. Kaiser, R. H. Osman and R. O. Dickau, “Analysis Guide for Variable Frequency Drive Operated Centrifugal Pumps," in Proceedings of the Twenty-Fourth International Pump Users Symposium, (2008).

[3] B. Schiavello, D. R. Smith and S. M. Price, "Abnormal Vertical Pump Suction Recirculation Problems Due to Pump-System Interaction," in Proceedings of the Twenty-First International Pump Users Symposium, (2004).

[4] J. C. Jo, "Fluid-Structure Interactions", Pressure Vessels and Piping Systems.

[5] ANSI/HI 9.8, 1998, "Pump Intake Design Standards," Hydraulic Institute, Parsippany, New Jersey.

[6] F. K. Benra, H.J. Dohmen, J. Pei, S. Schuster and B. Wan, J. Appl. Maths. 2011, (2011).

[7] D. R. Smith, G. M. Woodward, "Vibration Analysis of Vertical Pumps", in $15^{\text {th }}$ Turbomachinery Symposium, Texas, (1988).

[8] ANSI/HI 9.6.4, "Centrifugal and Vertical Pumps for Vibration Measurements and Allowable Values," Hydraulic Institute, Parsippany, New Jersey, (2000). 